\title{
Immobilization and removal of hazardous elements by geomaterials: the harder and the softer solutions
}

\section{G. Cruciani}

Department of Physics and Earth Sciences, University of Ferrara, Via Saragat 1, I-44122 Ferrara, Italy, cru@unife.it

Water and soil pollution by heavy metals and other hazardous compounds is a global problem threatening the entire biosphere and affecting the life of many millions of people around the world. For instance, approximately two million tons of industrial, sewage and agriculture waste are discharged every day into water, causing serious health problems and the death of many thousands of people every day on a worldwide basis [1]. Design and assessment of technologies for immobilization and removal of hazardous elements (HEs) is one of the highest priorities for environmental protection both in the industrialized and the developing countries [2].

The incorporation of HEs into crystalline and glassy silicate ceramics (the "harder" geomaterials) provides a perfect example to show that modelling the preferential distribution and the efficiency of immobilization of HEs in the crystal lattice and vitreous phase requires a detailed crystallographic knowledge at both the long- (XRD) and the short- (XAS) range (see Fig. 1) [3].

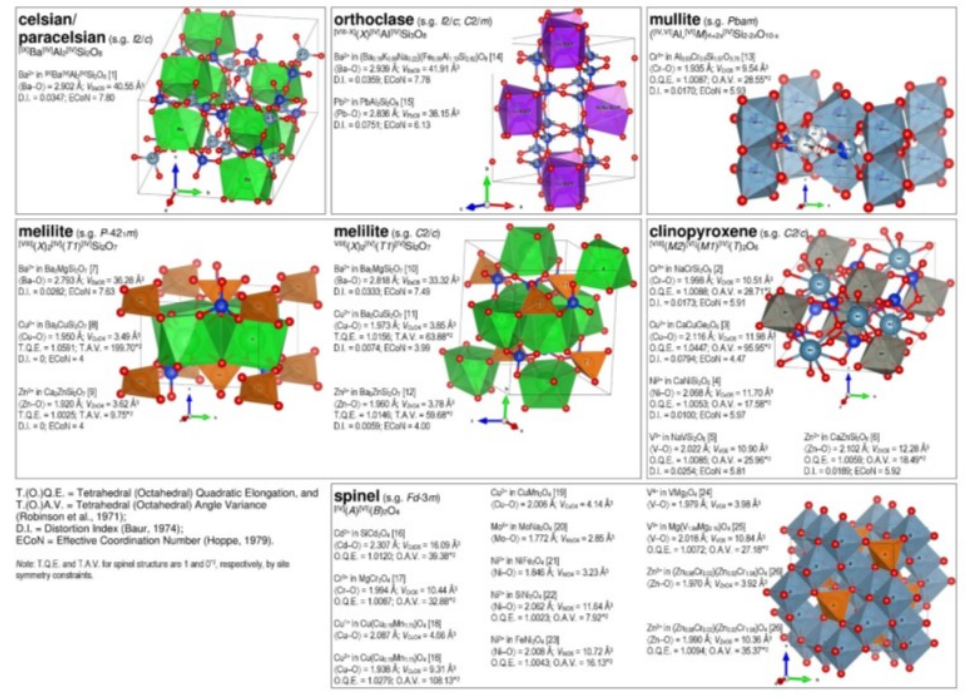

\begin{tabular}{|c|c|c|c|c|c|}
\hline Elem. & Valence state & $\begin{array}{l}\text { Oxygen } \\
\text { coordination }\end{array}$ & $\begin{array}{l}\text { Metal-oxygen } \\
\text { distance (A) }\end{array}$ & Complexes or oxyanions & Notes \\
\hline $\mathrm{Sb}$ & $\begin{array}{l}\mathrm{Sb}^{3 *} \text { predominant } \\
\mathrm{Sb}^{5+} \text { minor }\end{array}$ & $\begin{array}{l}\mathrm{Sb}^{3+}[3] \\
\mathrm{Sb}^{5+}[6]\end{array}$ & $\begin{array}{l}\mathrm{Sb}^{3}-01.938 \\
\mathrm{Sb}^{5}-01.964\end{array}$ & $\begin{array}{l}{\left[\mathrm{Sb}^{3} \cdot 0 \mathrm{O}\right] \mathrm{]} \text { likely in }} \\
\left.4\left[\mathrm{Sb}^{3} \cdot 0\right]\right] \text { rings }\end{array}$ & $\begin{array}{l}\mathrm{Sb}^{5}+\text { increasing with } \mathrm{O}_{2}, \mathrm{CaO} \\
\text { and glass basicity }\end{array}$ \\
\hline As & $\begin{array}{l}\mathrm{As}^{3 *} \text { predominant } \\
\mathrm{As}^{5 *} \text { minor }\end{array}$ & $\begin{array}{l}\mathrm{As}^{3 *}[3] \\
\mathrm{As}^{5 *}+[4]\end{array}$ & $\mathrm{As}^{3+}-01.78$ & {$\left[: A^{3} \cdot O_{3}\right]$} & 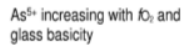 \\
\hline Ba & $\mathrm{Ba}^{2 *}$ & $\mathrm{Ba}^{2+}[12]$ & $\mathrm{Ba}^{2 *}-02.75-3.5$ & $\left.\left[A \mid{ }^{3}+O_{4}\right\}-B a^{2}-[A]^{3}+O_{4}\right]$ & $\begin{array}{l}\text { charge compensator of } \mathrm{AP} \text { - in } \\
\text { tetrahedral coordination }\end{array}$ \\
\hline $\mathrm{Cr}$ & $\begin{array}{l}\mathrm{Cr}^{3+} \text { predominant } \\
\mathrm{Cr}^{+6} \text { minor } \\
\mathrm{Cr}^{6} \text { minor }\end{array}$ & $\begin{array}{l}\mathrm{Cr}^{3}[6] \\
\mathrm{Cr}^{4}\left[\mathrm{r}^{4}[4]\right. \\
\mathrm{Cr}^{6+}[4]\end{array}$ & $\begin{array}{l}C^{3}+01.96 \\
C^{6}+01.72\end{array}$ & $\begin{array}{l}\mathrm{Cr}^{3+}-\mathrm{Cr}^{3+} \text { dimers } \\
\text { dominant for } \mathrm{Cr}_{2} \mathrm{O} 3>0.25 \% \\
{\left[\mathrm{Cr}^{6}+\mathrm{O}_{4}\right]^{2}}\end{array}$ & $\begin{array}{l}\text { Cret increasing with } t_{2} \text { and } \\
\text { glass basicity. Citre prevaling } \\
\text { with glass modifiers }>60 \%\end{array}$ \\
\hline $\mathrm{Cu}$ & $\begin{array}{l}\text { Cur predominant } \\
\mathrm{Cu}^{2+} \text { minor }\end{array}$ & $\begin{array}{l}\mathrm{Cu} \cdot[2] \\
\mathrm{Cu} \cdot[3] \text { minor } \\
\mathrm{Cu} \cdot[4] \text { minor }\end{array}$ & $\begin{array}{l}\mathrm{Cu}-0 \mathrm{O} 1.84[2] \\
\mathrm{Cu} u^{+}-01.88[3] \\
\mathrm{Cu} u^{+}-01.91[4]\end{array}$ & & $\begin{array}{l}\mathrm{Cu}^{2 *} \text { increasing with } 10 \text {, and } \\
\text { glass basicity. Coordination } \\
\text { number grows with } \mathrm{Cu}^{*} \%\end{array}$ \\
\hline $\mathrm{Pb}$ & $\mathrm{Pb}^{2+}$ & $\begin{array}{l}\mathrm{Pb}^{2+}+[3] \\
\mathrm{Pb}^{2+}[4] \text { minor }\end{array}$ & $\mathrm{Pb}^{2 *}-0[3] 2.24$ & & $\begin{array}{l}\mathrm{PbO}_{3} \text { and } \mathrm{PbO}_{4} \text { pyramidal } \\
\text { units in lead silicale glasses }\end{array}$ \\
\hline Mo & $\begin{array}{l}\text { Mot. predominant } \\
\text { Mot. minor }\end{array}$ & $\begin{array}{l}M_{0} 0^{6 *}[4] \\
\operatorname{Mo}^{4}[6]\end{array}$ & $\begin{array}{l}M_{0}^{6+}-0 \text { 1.75-1.78 } \\
M^{4}=0 \text { 2.01-2.02 }\end{array}$ & $\begin{array}{l}{\left[\mathrm{Mo}^{6}-\mathrm{O}_{4}\right]^{2}} \\
{\left[\mathrm{Mo}^{4-\mathrm{O}_{6}}\right]^{5}} \\
\text { molybdenyl } \mathrm{Mo}^{4 \cdot} \mathrm{O}^{2}+\end{array}$ & $\begin{array}{l}\text { Mo oxyanions are not } \\
\text { connected with the glass } \\
\text { tetrahedral network }\end{array}$ \\
\hline $\mathrm{Ni}$ & $\mathrm{Ni}^{2}+$ & $\begin{array}{l}N^{2 *} \cdot[4] \\
N^{2} \cdot[5] \text { minor }\end{array}$ & $\begin{array}{l}\mathrm{Ni}^{2}-01.95[4] \\
\mathrm{Ni}^{2}-02.00[5]\end{array}$ & & $\begin{array}{l}\mathrm{N}^{2^{2}} \cdot[4] \text { stabilized by } \mathrm{K} \\
\mathrm{N}^{2} \cdot[5] \text { by } \mathrm{Na}, \mathrm{Ca}, \mathrm{Mg}\end{array}$ \\
\hline Se & $\mathrm{Se}^{e^{6 *}}$ & $\mathrm{Se}^{64}[3]$ & $\mathrm{Se}^{6 \cdot-0} 1.69 \AA$ & & $\begin{array}{l}\text { fully inserted in the glass } \\
\text { network but in } \mathrm{Zn} \mathrm{glasses}\end{array}$ \\
\hline v & $\begin{array}{l}\mathrm{V}^{5+} \text { predominant } \\
\mathrm{V}^{4} \text { - minor }\end{array}$ & $\begin{array}{l}V^{55} \cdot[4] \\
V^{4} \cdot[5]\end{array}$ & $\begin{array}{l}V^{5}-01.69-1.70 \\
V^{4}-01.90\end{array}$ & {$\left[\mathrm{~V}^{5} \cdot \mathrm{O}_{4}\right]^{3}$} & V*s scarce \\
\hline $\mathrm{Zn}$ & $\mathrm{Zn}^{2+}$ & $\left.2 n^{2 *}+4\right]$ & $2 \mathrm{n}^{2 *}-0$ 1.95-1.96 & & glass network tormer \\
\hline
\end{tabular}

Figure 1. On the left: main crystalline phases that can form during firing of silicate ceramics with structural parameters of sites that can accommodate HEs. On the right: HEs hosted in natural and synthetic aluminosilicate melts and glasses [3].

Concerning the application of "softer" geomaterials, crystallographic knowledge of the zeolite structures proved successful for removal of heavy metals and VOCs from contaminated water [4] and for the clean-up of water polluted with antibiotics [5]. Simultaneous toxic metal uptake and bacteria disinfection from aqueous solution was achieved using well characterized nanocomposites of both the softer (zeolite) and the harder (e.g. $\mathrm{Fe}_{3} \mathrm{O}_{4}$ ) geomaterials [6].

[1] Bolisetty S., Peydayesh M. \& Mezzenga R. (2019). Chem. Soc. Rev. 48,463.

[2] Joseph L., Jun B-M., Flora J.R.V., Park C.M. \& Yoon Y. (2019). Chemosphere. 229, 142.

[3] Ardit M., Zanelli C., Conte S., Molinari C., Cruciani G. \& Dondi M. (2021). Submitted to J. Hazard. Mater.

[4] Braschi, I., Blasioli, S., Buscaroli, E., Montecchio, D. \& Martucci, A. (2016) J. Environ. Sci. (China) 43, 302

[5] Mancinelli M., Arfè A., Martucci A., Pasti L., Chenet T., Sarti E., Vergine G. \& Belviso C. (2020) Processes 8, 1519.

[6] Zendehdel, M., Ramezani, M., Shoshtari-Yeganeh, B., Cruciani, G. \& Salmani, A. (2019) Environ. Technol. 40, 3689.

Keywords: hazardous elements; geomaterials; immobilization

I wish to acknowledge my principal co-workers on this topic: Matteo Ardit \& Annalisa Martucci (University of Ferrara, Italy); Michele Dondi, Sonia Conte, Chiara Molinari \& Chiara Zanelli (CNR-ISTEC, Italy); Mojgan Zendehdel (University of Arak, Iran).

Acta Cryst. (2021), A77, C646 